\title{
Cationic Emulsion-Based Artificial Tears as a Mimic of Functional Healthy Tear Film for Restoration of Ocular Surface Homeostasis in Dry Eye Disease
}

\author{
Philippe Daull, ${ }^{1}$ Mourad Amrane, ${ }^{1}$ Dahlia Ismail, ${ }^{1}$ Georgi Georgiev, ${ }^{2}$ Lukasz Cwiklik, ${ }^{3}$ \\ Christophe Baudouin, ${ }^{4}$ Andrea Leonardi, ${ }^{5}$ Gerhard Garhofer, ${ }^{6}$ and Jean-Sebastien Garrigue ${ }^{1}$
}

\begin{abstract}
Dry eye disease (DED) is a complex multifactorial disease that affects an increasing number of patients worldwide. Close to $30 \%$ of the population has experienced dry eye (DE) symptoms and presented with some signs of the disease during their lifetime. The significant heterogeneity in the medical background of patients with DEs and in their sensitivity to symptoms renders a clear understanding of DED complicated. It has become evident over the past few years that DED results from an impairment of the ocular surface homeostasis. Hence, a holistic treatment approach that concomitantly addresses the different mechanisms that result in the destabilization of the tear film (TF) and the ocular surface would be appropriate. The goal of the present review is to compile the different types of scientific evidence (from in silico modeling to clinical trials) that help explain the mechanism of action of cationic emulsion (CE)-based eye drop technology for the treatment of both the signs and the symptoms of DED. These CEbased artificial tear (AT) eye drops designed to mimic, from a functional point of view, a healthy TF contribute to the restoration of a healthy ocular surface environment and TF that leads to a better management of DE patients. The CE-based AT eye drops help restore the ocular surface homeostasis in patients who have unstable TF or no tears.
\end{abstract}

Keywords: dry eye, tear film lipid layer, artificial tear, cationic emulsion, polar lipid

\section{Introduction}

A $\mathrm{N}$ EFFICIENT STRATEGY-AND probably the most reasonable one-for the treatment of multifactorial diseases is to simultaneously modulate the different key factors identified as having a causative role in the disease. Hence, a good understanding of the physiopathology of multifactorial diseases with an intention-to-treat approach is of utmost importance. Dry eye disease (DED) has been described as a multifactorial disease ${ }^{1-3}$ explained by a vicious-circle-like mechanism that perpetuates/exacerbates the disease process. ${ }^{4}$ The TFOS DEWS II 2017 workshops on dry eye (DE) pathophysiology ${ }^{5}$ established a revised DED definition that summarizes the main characteristics of the disease and lists its major contributing factors. The revised TFOS DEWS II definition of DE states: "Dry eye is a multifactorial disease of the ocular surface characterized by a loss of homeostasis of the tear film, and accompanied by ocular symptoms, in which tear film instability and hyperosmolarity, ocular surface inflammation and damage, and neurosensory abnormalities play etiological roles." ${ }^{5}$ Further, the Asian Dry Eye Society (ADES) emphasized the role of tear film (TF) instability in DED and of TF-oriented therapy (TFOT) approaches for the management of DE patients. ${ }^{6,7}$

In DED, the stable state of equilibrium (i.e., homeostasis) of the ocular surface and the TF is impaired. For homeostasis

\footnotetext{
${ }^{1}$ Novagali Innovation Center, Santen SAS, Evry, France.

${ }^{2}$ Biointerfaces and Biomaterials Laboratory, Department of Optics and Spectroscopy, School of Optometry, Faculty of Physics, St. Kliment Ohridski University of Sofia, Sofia, Bulgaria.

${ }^{3}$ J. Heyrovsky Institute of Physical Chemistry, Czech Academy of Sciences, Prague, Czech Republic.

${ }^{4}$ CHNO des Quinze-Vingts, IHU FOReSIGHT, INSERM-DGOS CIC 1423, Paris, France \& Sorbonne Universités, INSERM, CNRS, Institut de la Vision, Paris, France.

${ }^{5}$ Department of Neuroscience, Ophthalmology Unit, University of Padua, Padua, Italy.

${ }^{6}$ Department of Clinical Pharmacology, Medical University of Vienna, Vienna, Austria.

(C) Philippe Daull et al., 2020; Published by Mary Ann Liebert, Inc. This Open Access article is distributed under the terms of the Creative Commons Attribution Noncommercial License (http://creativecommons.org/licenses/by-nc/4.0/) which permits any noncommercial use, distribution, and reproduction in any medium, provided the original author(s) and the source are cited.
} 
to be restored, it is necessary to alleviate the signs and symptoms of DE and concomitantly improve the different, interdependent elements constituting the ocular surface environment. Indeed, the correction of only one of these interdependent elements cannot result in the restoration of a stable state of equilibrium, and although it can "slow down" the vicious circle of DE it cannot interrupt or correct all of the disease processes in DED. For example, supplementing the ocular surface with aqueous-based eye drops or gel-like lubricants may help reduce the symptoms temporarily but will not be effective over a long period since they do not restore the steady-state equilibrium of the ocular surface but instead shift it toward an excess of water; this excess water is ultimately lost over time, with a return to an unstable and unfavorable state requiring frequent re-instillations.

Thus, to restore this state of equilibrium and consequently a healthy environment for the eye, the following issues must be dealt with simultaneously: (1) TF instability; (2) TF water-phase hyperosmolarity; (3) ocular surface inflammation; (4) corneal and conjunctival epithelium damage; and (5) neurosensory abnormalities.

\section{The Cationic Emulsions for Dry Eye Management}

Cationic emulsion (CE)-based artificial tear (AT) eye drops $^{8,9}$ have recently developed lipid-containing ATs (Cationorm ${ }^{\circledR}$, Cationorm Pro, Santen) ${ }^{10}$ that besides CE-based drug carriers (Ikervis ${ }^{\circledR}$, Santen) ${ }^{11,12}$ have been designed for the management of the clinical signs and symptoms of DED as well as the management of vernal keratoconjunctivitis in children (Verkazia ${ }^{\circledR}$, Santen). ${ }^{13}$ The present review compiles and discusses the findings from extended in silico, in vitro, and in vivo studies of CEs. It aims at (1) exploring and gaining insight into the mechanism of action of CEs, (2) explaining how CEs are able to improve the ocular surface condition of $\mathrm{DE}$ patients by simultaneously modulating/correcting the different etiological factors of DE, as presented in the TFOS DEWS II definition, ${ }^{5}$ and (3) thereby demonstrating that a holistic approach - and the treatment of the ocular surface as a whole-is effective for the management of these patients.

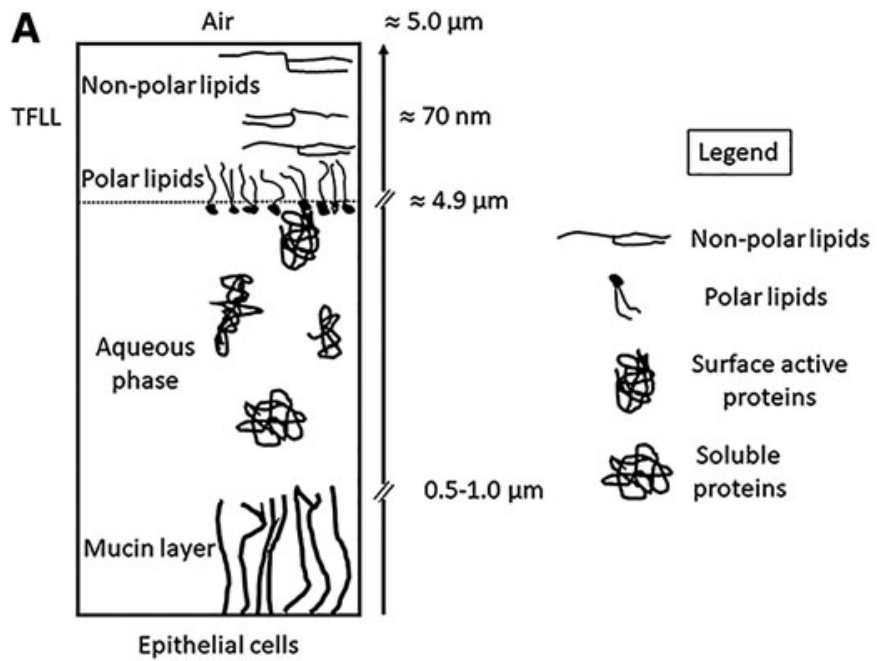

\section{Improved TF stability with the CEs}

The TF is composed of 3 different layers (Fig. 1A, B and Table 1): a tear film lipid layer (TFLL), that is, the outermost layer of the TF, an aqueous phase that contains surface-active proteins and soluble mucins, and the mucin layer/glycocalyx that is directly adjacent to the corneal and conjunctival epithelial cells. ${ }^{14}$ The TFLL plays an important role in TF stabilization. ${ }^{15}$ It has been demonstrated that a thicker TFLL results in more stable TF with a longer tear break-up time, whereas thinner and more heterogeneous TFLLs are associated with less stable TF. ${ }^{16}$ The TFLL contains mainly nonpolar lipids (>90\%-92\%) and up to $8 \%$ amphiphilic polar lipids [6.0\% phospholipids, $1.8 \%$ sphingolipids, and 3\% (O-acyl)- $\omega-$ hydroxy fatty acids (OAHFAs)], ${ }^{17-19}$ which predominantly play a surfactant role. More than 93\% of TFLL lipids (all nonpolar lipids and OAHFA) are secreted by the meibomian glands. Thus, a dysfunction of the meibomian glands results in quantitative and qualitative alterations of the TFLL and in the development of DED. ${ }^{20}$ Indeed, up to $80 \%$ of DE patients have meibomian gland dysfunction (MGD). It is, therefore, important to compensate for the nonpolar and polar lipid loss observed in these patients to restore the thickness and stability of the TFLL-hence the development of lipid-containing (Fig. 1B, and Table 1) eye drops. ${ }^{21}$

Polar lipids are crucial to stabilize the interface between the TFLL and the aqueous phase of the TF. Polar lipids are amphiphilic molecules possessing hydrophobic moiety (i.e., with a high affinity for oily environments) and at the same time a hydrophilic head (i.e., with a high affinity for aqueous environments). This renders them surface active (i.e., with surfactant properties), which enables the polar lipids to be distributed naturally at the oil-water interface acting as a TFLL spreading agent and stabilizing the tear surface by segregating the hydrophobic molecules from water molecules. ${ }^{1522,23} \mathrm{Al}-$ though the contribution of exogenous non-meibomian polar lipids to the TFLL remains unclear, it was clinically demonstrated that OAHFA (the endogenous surfactant of meibum) deficiency correlates with the severity of DE. ${ }^{24}$

Recently, Cwiklik and collaborators ${ }^{25}$ demonstrated, using in silico coarse-grain molecular dynamic simulations, that polar lipid deficiency generates unfavorable water-

B

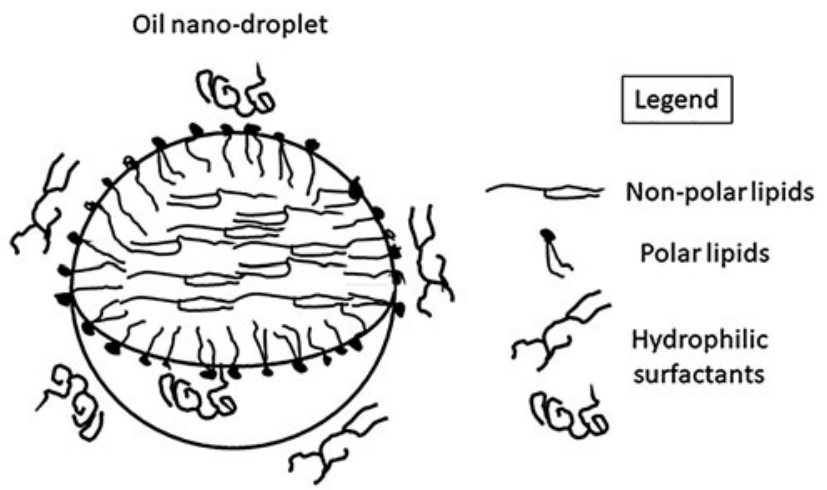

FIG. 1. Comparison of the structure of a healthy tear film (A) and the CEs (B). CE, cationic emulsion; TFLL, tear film lipid layer. 
Table 1. Quantitative Composition of a Healthy Tear Film and the Cationic Emulsions

\begin{tabular}{|c|c|c|}
\hline \multirow[b]{2}{*}{ TF structure } & Healthy natural TF & Cationic emulsions \\
\hline & Composition & Composition \\
\hline \multicolumn{3}{|l|}{ Lipid layer $(0.015-0.160 \mu \mathrm{m})$} \\
\hline Apolar lipids & $\begin{array}{l}\text { Wax ester, cholesterol esters, TGs } \\
\quad(>90 \% \text { of total lipids })^{14,17-19}\end{array}$ & Mineral oils $(1 \%)$ or MCT $(2 \%)^{8,9}$ \\
\hline Polar lipids & $\begin{array}{l}\text { OAHFA, PC, PE, lysoPC, SM, etc. } \\
\quad(\sim 8 \% \text { of total lipids })^{14,17-19}\end{array}$ & $\mathrm{CKC}(0.002 \%-0.005 \%)^{8,9}$ \\
\hline \multicolumn{3}{|c|}{ Low-viscosity aqueous layer $(\sim 4 \mu \mathrm{m})$} \\
\hline Water & Water $(\sim 98.2 \%)$ & Purified water $(96 \%-98 \%)^{8,9}$ \\
\hline Serum \& tear specific proteins & Surface-active lipocalin $(2 \mathrm{mg} / \mathrm{mL})$ & Poloxamer $(0.1 \%)$ \\
\hline$(6-10 \mathrm{mg} / \mathrm{mL})$ & $\begin{array}{l}\text { \& soluble mucins }{ }^{14} \\
\text { (help in TF spreading) }\end{array}$ & $\begin{array}{l}\text { Tyloxapol }(0.3 \%) \text { (help in TF } \\
\text { spreading) }\end{array}$ \\
\hline Metabolites \& electrolytes & $\begin{array}{l}\text { Glucose, lactate, etc. \& Various salts } \\
\quad \text { (tonicity: iso-osmolar }<308 \mathrm{mOsmol} / \mathrm{L})^{14}\end{array}$ & $\begin{array}{l}\text { Glycerol (osmoprotectant) } \\
\quad(1.6 \%-2.25 \%) \& \mathrm{NaCl} \\
\quad \text { (tonicity: } 190-270 \mathrm{mOsml} / \mathrm{L})^{8,9}\end{array}$ \\
\hline \multicolumn{3}{|l|}{ Gel-like mucin layer $(2.5-5 \mu \mathrm{m})$} \\
\hline Glycosylated proteins & $\begin{array}{l}\text { Epithelial cell-bound sugar-rich } \\
\text { glycosylated proteins }{ }^{4}\end{array}$ & - \\
\hline
\end{tabular}

CKC, cetalkonium chloride; LysoPC, lysophosphatidylcholines; MCT, medium-chain triglyceride; OAHFA, (O-acyl)- $\omega$-hydroxy fatty acid; PC, phosphatidylcholines; PE, phosphatidylethanolamines; SM, sphingomyelins; TF, tear film; TG, triglycerides.

nonpolar lipid contact leading to destabilization of the TF. The CE, with its positively charged amphiphilic molecule [cetalkonium chloride (CKC); i.e., a polar lipid], and the surfaceactive water-based excipients (poloxamer 188 and tyloxapol) help compensate for the deficiency of polar lipids and stabilize the lipid film. ${ }^{25}$ Surface chemistry studies using Langmuir troughs and Brewster angle microscopy with the $\mathrm{CE} \mathrm{AT}^{26}$ and CE-based drug products ${ }^{27}$ have demonstrated that CEs interact favorably with meibomian gland secretions (i.e., TFLL) and stabilize the lipid film by increasing its elasticity. Indeed, as shown through in silico modeling, CKC is stably incorporated in the TFLL at the aqueous interface (Fig. 2A), and it can compensate for a moderate deficiency of polar lipids. ${ }^{28,29}$ The concomitant action of poloxamer and tyloxapol, 2 surfaceactive hydrophilic compounds that are adsorbed at the TFLL interface and slightly penetrate the lipid film, synergistically contribute to the stabilization of the TFLL, ${ }^{25}$ even though they are eventually squeezed out of the TFLL interface after repeated cycles of eye blinking. ${ }^{26}$

Interestingly, the polar lipid present in the CEs (i.e., CKC) also harbors a positive charge. This positive charge has various important roles in the stabilization of the TFLL and its interface. It helps position the polar lipid at the interface of the TFLL with the positive charge facing the aqueous phase. Through electrostatic interactions with the positively charged moieties exposed to water, the negatively charged soluble proteins (mucins, lysozyme) present in the aqueous phase of the TF are adsorbed at the interface with the TFLL. ${ }^{30}$ It is likely that such interactions add to the positive effect of $\mathrm{CKC}$, since adsorbed proteins help reduce the surface tension of the TFLL and further improve the overall stability of the TF.

The same basic physical principles that have driven the choice of polar lipid (CKC) and hydrophilic surfactant (poloxamer and tyloxapol) for long-term stability ${ }^{8}$ of the CEs (3-year shelf-life in their dropper) also account for the stabilization of the TFLL after CE instillation on the ocular surface of DE patients (Table 2). The nonpolar lipids (Fig. 2B, C) provided by the CEs were selected for their excellent miscibility with the TFLL long-chain lipids (light and heavy min- eral oil) or for their ability to spread over the TFLL at the TFLL-air interface (medium-chain triglyceride) to improve the thickness of the TFLL. ${ }^{26,27,31,32}$ The favorable interactions of CE lipophilic constituents (nonpolar lipids and CKC) with meibum are believed to facilitate their stable incorporation into the TFLL, which, in view of the slow turnover rate of the TFLL $(0.93 \pm 0.36 \% / \mathrm{min}),{ }^{33}$ is expected to result in an extended residence time and therapeutic efficacy of the $\mathrm{CE}$ at the ocular surface. The effect of CE instillation on TFLL is observable $1 \mathrm{~h}$ after instillation (Fig. 2D).

This fundamental surface chemistry and in silico molecular dynamics simulations research on CEs and their molecular components (polar lipids and hydrophilic surface-active excipients) explain from a mechanistic physicochemical point of view the improvement in TF break-up time observed in clinical trials, for both the CE ATs ${ }^{10,34}$ and CE drug products. ${ }^{11,35}$ In addition, a comparison of $\mathrm{CE}$ versus a polyvinyl alcohol and povidone-based lubricant demonstrated that a statistically significant improvement in tear break-up time was observed on day $28(+2.0$ vs. $+0.5 \mathrm{~s} ; P=0.0349)$ in MGD patients treated with the $\mathrm{CE}$, hence confirming that the $\mathrm{CE}$ may act on the TF stability. ${ }^{34}$ The lipid layer thickness was also found to be increased after the instillation of CE in patients ${ }^{32}$ and in healthy volunteers. Optical coherence tomography-based TFLL thickness increased on average by $34 \%$ after administration of the CE AT. The maximum increase $(60.8 \% \pm 28 \%)$ occurred 30 min after instillation. ${ }^{31}$

\section{Reduced hyperosmolarity and its associated cellular stress with the CEs}

Tear hyperosmolarity can induce cell damage that results in the apoptosis of corneal and conjunctival epithelial cells as well as goblet cells and in altered mucin production, 36,37 which, in turn, exacerbate TF instability, thus leading to more tear hyperosmolarity. This tear hyperosmolarity was described as one of the causes of DED and the associated inflammation of the ocular surface..$^{5,36,38,39}$ The typical values of tear osmolarity in healthy patients range from 290 


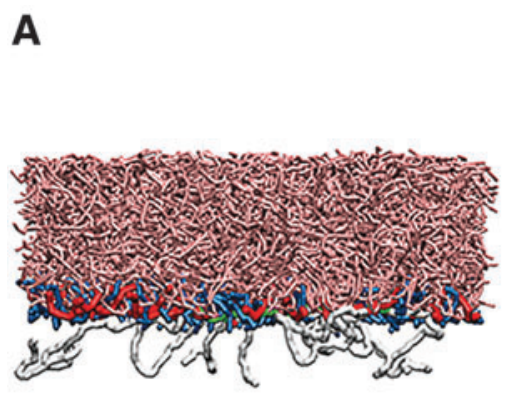

Side view of the lipid film

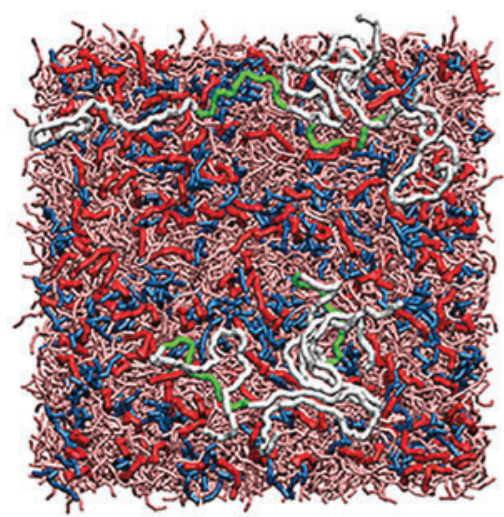

Bottom view of the lipid film

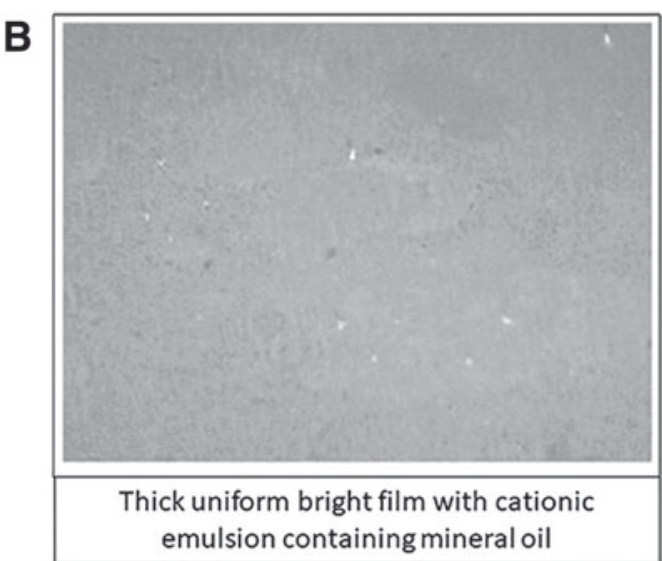

Non-polarlipid: pink

Polar lipid: blue

CKC: red

Poloxamer: white \& green
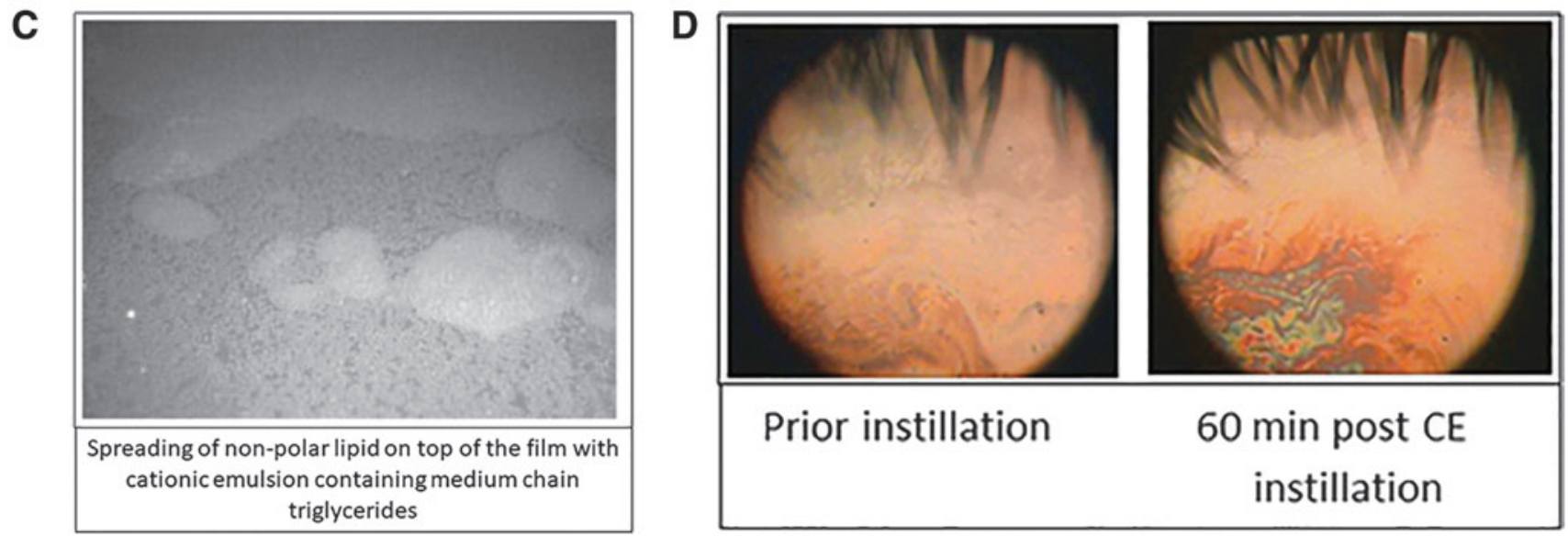

FIG. 2. Coarse-grain molecular dynamic simulations (side and bottom-up views of the lipid film) of the incorporation of CKC and poloxamer in lipid film with polar lipid deficiency. Non-polar lipids, pink; polar lipids, blue; CKC, red; Poloxamer, white and green (A). Brewster angle microscopy images of the excellent miscibility of mineral oil (B) with MGS, and (C) spreading of medium-chain triglyceride over MGS. (D) Specular microscopy images of TFLL before and after instillation of $\mathrm{CE}$ at the ocular surface; field of view is $\sim 3 \mathrm{~cm}^{2}$ (adapted from Georgiev et al.). ${ }^{15} \mathrm{CKC}$, cetalkonium chloride; MGS, meibomian gland secretion.

to $308 \mathrm{mOsm} / \mathrm{L}^{40-42}$ A tear osmolarity threshold of $308 \mathrm{mOsm} / \mathrm{L}$ has been proposed to distinguish between patients with early signs of DE and patients with normal eyes. $^{37,41}$ Hyperosmolarity values not only of $>380 \mathrm{mOsm} / \mathrm{L}$ (and up to $441 \mathrm{mOsm} / \mathrm{L}$ ) but also of normal to hypoosmolarity can be found in patients with severe DE. ${ }^{43,44}$

A temporary decrease in hyperosmolarity (as a transient "osmocorrection") can be achieved through the use of hypo-osmolar to iso-osmolar eye drops. ${ }^{11,45}$ In that case, the instillation of eye drops results, by a simple dilution of the hyperosmolar tear, in a decrease in tear hyperosmolarity (toward its normalization) that lasts for a couple of minutes and is related to the (short) turnover of the aqueous phase of the tears. ${ }^{33} \mathrm{In}$ deed, with a turnover rate (in healthy adults) of $10.3 \pm 3.7 \% / \mathrm{min}$ for the tear aqueous phase, all of the water volume and watersoluble solutes [including water-soluble polymers like hyaluronic acid (HA) or carboxymethyl cellulose] instilled with the eye drops are eliminated from the TF in $15-20 \mathrm{~min}^{33}$

This strategy is used by many commercially available water-based eye drop solutions, and it is applied to the CEs too. The water phase of the $\mathrm{CE}$ eye drops, which represents $90 \%-96 \%$ of the volume, is hypo-osmolar $(190 \mathrm{mOsm} / \mathrm{L})$ in the CE ATs and slightly hypo-osmolar to iso-osmolar $(270 \mathrm{mOsm} / \mathrm{L})$ in $\mathrm{CE}$ drug products or in second-generation CE ATs. Adding small-molecular-weight osmoprotectants ${ }^{46}$ to the eye drops (water phase), which can on instillation enter epithelial cells and stabilize their volume (by retaining water molecules), will have a more sustained protective effect on cell survival and their resistance to the osmotic stress generated by hyperosmolarity. ${ }^{47,48}$ For that purpose, the CE eye drops contain glycerol $(1.6 \%-2.25 \%)$ as an osmoprotecting agent. ${ }^{11,49}$

\section{Management of ocular surface inflammation with the CEs}

Hyperosmolarity of the $\mathrm{TF}$ is a strong trigger of inflammatory cascades 46,50 in corneal and conjunctival epithelial cells, which translates clinically into epithelial damage and mucin coverage deficiency, as evidenced, for example, via corneal fluorescein staining (CFS) tests. ${ }^{51}$ Based on their ability to 


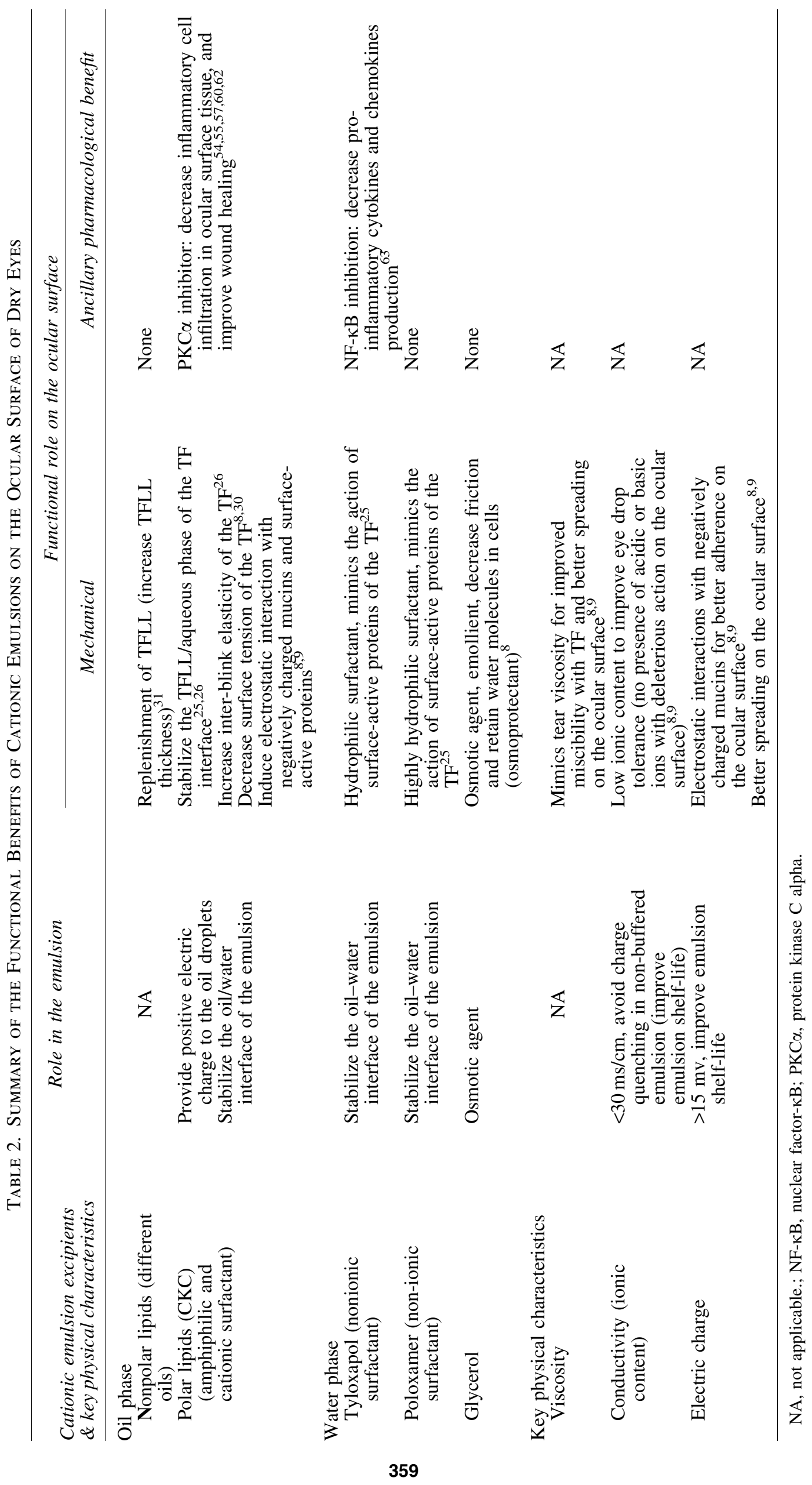


reduce hyperosmotic stress, CEs might help in the management of ocular surface inflammation. ${ }^{52}$ However, the hypotonicity of the eye drops alone may not be sufficient to fully explain the improvement seen in CFS scores.

The Nosika trial ${ }^{10}$ compared 2 hypo-osmolar (i.e., hypotonic) eye drops - the CE AT $(190 \mathrm{mOsm} / \mathrm{L})$ and an HA-based AT $(0.18 \%$ HA, $150 \mathrm{mOsm} / \mathrm{L})$-in patients with mild-to-moderate DE. As early as 7 days after treatment initiation, $22.7 \%$ of the patients treated with the CE AT reached a CFS score of 0 (i.e., complete healing of the cornea), whereas only $9.7 \%$ of the patients treated with the HA-based AT reached a CFS score of 0 . It was only at the end of the treatment period ( 3 months) that $22.0 \%$ of the patients treated with the HA-based AT had also reached a CFS score of 0. By contrast, at completion of the study, $31.8 \%$ of the patients treated with the CE AT had reached a CFS score of 0 .

Interestingly, a similar efficacy was observed in the Sansika trial, ${ }^{11,35}$ in which patients with severe DE (CFS $\geq 4$ in the Oxford grading score, which has a maximum of 5 , at inclusion) were treated with the CE AT (i.e., the CE empty vehicle). At 6 months, $48.1 \%$ of the patients treated with the CE AT experienced a 2-grade reduction in CFS score, and among them $14.4 \%$ saw their CFS score decrease by 3 grades (CFS score decreased from 4 to 1 ). In a mouse model of DED, a similar improvement in CFS scores was observed on treatment with the CE AT ${ }^{52,53}$ Interestingly, in this mouse model of DED, the CE AT was as effective at reducing CFS scores as $1 \%$ methylprednisolone, a potent glucocorticoid used for its anti-inflammatory properties.

Further in vivo and in vitro studies were performed to explore the underlying anti-inflammatory effects of the CEs that may contribute to their efficacy profile observed in animal models and in clinical trials.

A rat model of corneal epithelial abrasion was used to generate superficial lesions and inflammation in the cornea. On treatment with the $\mathrm{CE}$, it was observed that the healing process of the corneal epithelium was accompanied by a significant reduction in the count of infiltrating inflammatory cells (Fig. 3). ${ }^{54}$ When the CE AT was compared with other commercially available ATs (Systance Balance ${ }^{\circledR}$, Optive ${ }^{\circledR}$, and Vismed $^{\circledR}$ ) in this model, the cornea was found to heal similarly in all treatment groups, but the CE AT-treated corneas showed the lowest number of infiltrating inflammatory cells in the corneal stroma. ${ }^{55}$ The CE AT was also the only AT to have a marked direct anti-inflammatory effect on the secretion of proinflammatory interleukin (IL)-6 and IL-8 from human corneal epithelial (HCE-2) cells stimulated by a lipopolysaccharide (LPS) challenge. ${ }^{55}$ This direct anti-inflammatory action of the CEs was further confirmed in peripheral blood mononuclear cells (PBMCs; stimulated with anti-CD3/antiCD28 or LPS), and in CD4+ T lymphocytes (TCD4; stimulated with anti-CD3/anti-CD28 antibodies). ${ }^{56,57}$ The CEs decreased the expression of interferon (IFN)- $\gamma$, IL$17 \mathrm{~A}, \mathrm{CXCL}-9$, and tumor necrosis factor (TNF)- $\alpha$ genes in LPS-stimulated PBMCs, and they decreased the expression of THBS1 and CCL2 genes in LPS-stimulated HCE-2 cells. The CEs also inhibited the secretion of IL-17 (from anti-CD3/anti-CD28-stimulated TCD4), TNF $\alpha$, IFN- $\gamma$, and IL-2 (from anti-CD3-/anti-CD28-stimulated PBMCs) as well as IL-6 and IL-8 (from LPS-stimulated HCE-2). ${ }^{57}$

After this rather unexpected direct anti-inflammatory effect of the CEs, it was interesting to further explore the mechanism of anti-inflammatory activity and identify the

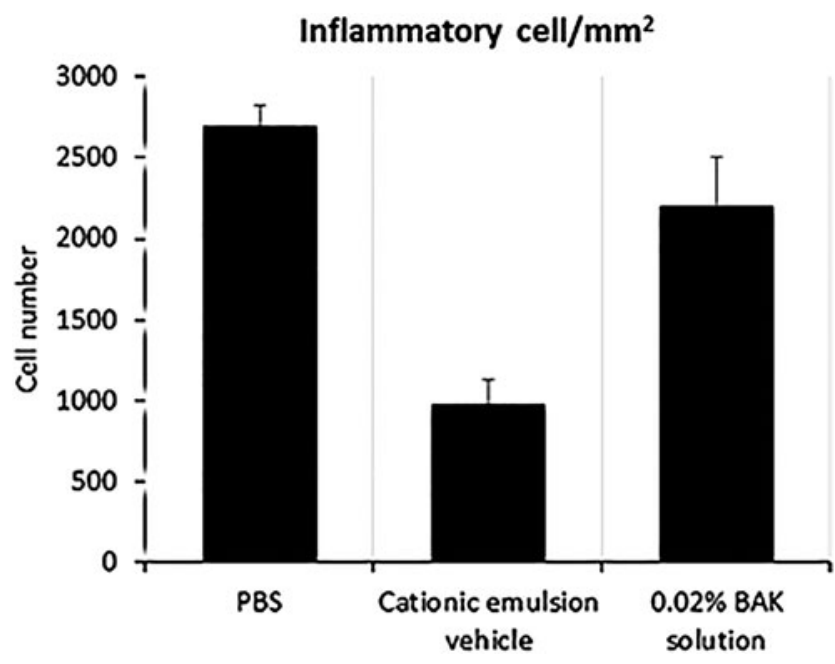

FIG. 3. Inflammatory cell counts in the cornea of rat treated with the CE ATs (adapted from Liang et al.). ${ }^{60}$ ATs, artificial tears.

excipient(s) supporting it. Quite surprisingly, the cationic excipient, $\mathrm{CKC}$, used to bring the positive charge to the oil droplets within the emulsion ${ }^{8,9}$ was demonstrated to be a specific inhibitor of protein kinase $\mathrm{C}$ alpha $(\mathrm{PKC} \alpha) .{ }^{57} \mathrm{CKC}$ is a lipophilic surfactant from the benzyl alkyl quaternary ammonium chloride family. Although it is the most hydrophobic subcomponent of the benzalkonium chloride (BAK) mixture-which explains its improved safety profile when formulated in emulsion ${ }^{8,9,58-61}$ versus the benzyl alkyl quaternary ammoniums chloride with shorter aliphatic chains (12 or 14 carbons) $)^{8,9}$-it was not anticipated that CKC, through its modulation of $\mathrm{PKC} \alpha$, could be even partly responsible for the good clinical performance of the CEs. ${ }^{10,11,35}$ Indeed, Pflugfelder and collaborators demonstrated in a PKC $\alpha$ knock-out mouse the benefits that $\mathrm{PKC} \alpha$ "inhibition" has on corneal inflammation and its healing process. ${ }^{62}$

When formulated in CEs, CKC does not harbor any of the detrimental side effects of the shorter BAK quaternary ammonium compounds, but instead offers the benefits of modulating $\mathrm{PKC} \alpha$ for the management of inflammation and the improvement of wound-healing processes in DE patients in addition to mechanically stabilizing the TFLL. The CKC in the CE represents $\sim 0.25 \%$ of the oily phase, thus ensuring supplementation of physiologically optimal amounts of cationic compounds to the ocular surface. In addition, another excipient present in CEs-tyloxapol, a water-soluble surfactant-was demonstrated to have anti-inflammatory activity that may contribute to the efficacy of CEs. ${ }^{57}$ Indeed, it was previously described that tyloxapol is able to suppress nuclear factor- $\kappa \mathrm{B}$ $(\mathrm{NF}-\kappa \mathrm{B})$ activation in monocytic cells, ${ }^{63}$ that it has an inhibitory effect on cytokine production, ${ }^{64}$ and prevents macrophages from reacting to endotoxin. ${ }^{65}$ Tyloxapol $(0.3 \%)$ alone, formulated as an emulsion of medium-chain triglycerides, was able to reduce the secretion of IL- 6 and IL- 8 by $21 \%$ and $50 \%$, respectively, in LPS-induced HCE-2 cells. 57

\section{Improvement of ocular surface tissue damage with the CES}

Ocular surface damage, that is, corneal and/or conjunctival epithelium lesions, is generally observed in DED patients 


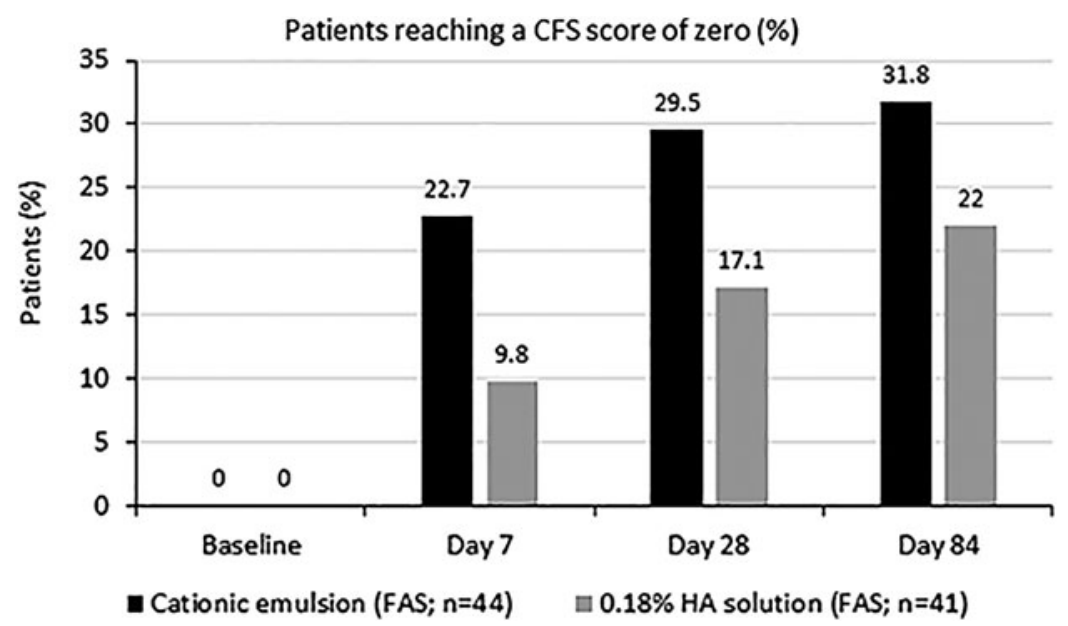

FIG. 4. Percentage of corneal clearing on treatment with the CE AT and HA-based eye drop in patients with mild-to-moderate DED with CFS scores up to 2 at study inclusion. CFS, corneal fluorescein staining; DED, dry eye disease; HA, hyaluronic acid. via staining techniques by using fluorescein or lissamine green dyes. They allow for the establishment of scores that measure the severity of the lesions: The higher the score, the more severe the tissue damage. The CFS score for corneal damage and the van Bijsterveld/lissamine green score for conjunctival injury are the most commonly used tools to assess ocular surface tissue damage. ${ }^{66}$ The use of ATs/tear substitutes was demonstrated to reduce CFS scores in DED patients with mild-to-moderate signs of the disease. This was observed in the Nosika trial comparing CE ATs with an HAbased AT (Fig. 4). Simple lubrication of the ocular surface can be sufficient, to a certain extent, for achieving a clinically significant decrease in CFS scores. Thus, the restoration of a healthy environment of the ocular surface contributes toward decreased tissue damage.

It is interesting to note that the stabilization of the TFLL and the TF with the CE AT has "stronger" effects on the healing of the cornea (Fig. 4) than a jellified aqueousbased HA solution. ${ }^{10}$ Besides, the anti-inflammatory effect of the CE AT observed in animal models may also contribute to the improvement of the CFS score. ${ }^{55,57}$ However, in more severe DED conditions, for example, for patients with CFS scores of $\geq 4$, classic aqueous-based ATs were not sufficient to improve the ocular surface tissue damage. This was observed in a large clinical trial (San- sika study) where patients at inclusion had a mean daily number of 8 aqueous-based AT instillations ${ }^{11}$ and still showed significant corneal damage with a CFS score of $\geq 4$ at the beginning of the study, thus clearly demonstrating the lack of efficacy of lubricating ATs (aqueous-based or viscosifying agent-containing eye drops) for the treatment of severe DED.

Interestingly, on top of these classic aqueous-based ATs, the addition of just 1 drop per day of the CE led to a 2-grade decrease in CFS score in $48.1 \%$ of the patients at 6 months $^{11} ; 4.4 \%$ of the patients even experienced complete healing of their cornea, with a CFS score of 0 (Fig. 5). Only $20.5 \%$ of these patients with severe DED experienced no improvement after the addition of 1 drop per day of CE.

As previously discussed, it was demonstrated in a mouse model of severe DED that the CE AT was as effective as, if not better than, $1 \%$ methylprednisolone in reducing corneal lesions. $^{52}$ The CE AT was also shown to improve the elasticity of the TFLL, by supplementing the ocular surface with polar lipids (i.e., CKC), and the thickness of the TFLL, through its delivery of nonpolar lipids, with the overall effect of improving the stability of the TF. ${ }^{26}$ The concerted mechanical and pharmacological effects of the excipients present in the CEs might explain the benefits observed on the wound healing after the instillation of CE.
A

CFS change over time

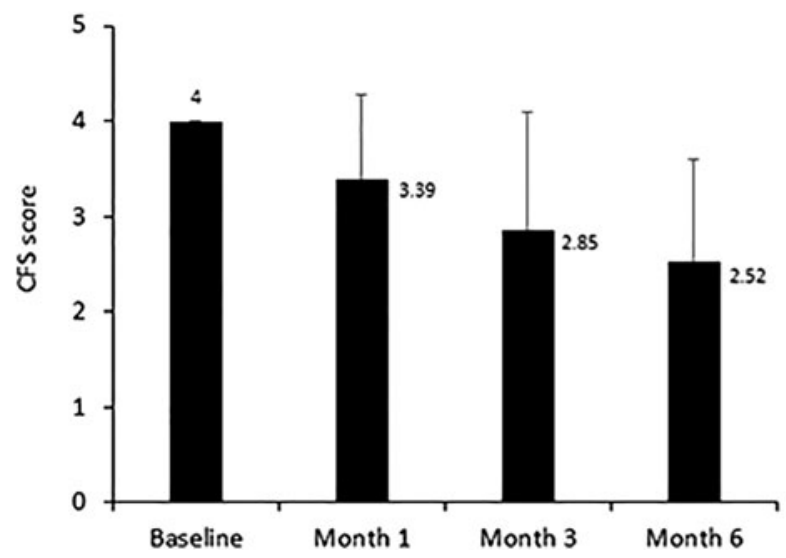

B

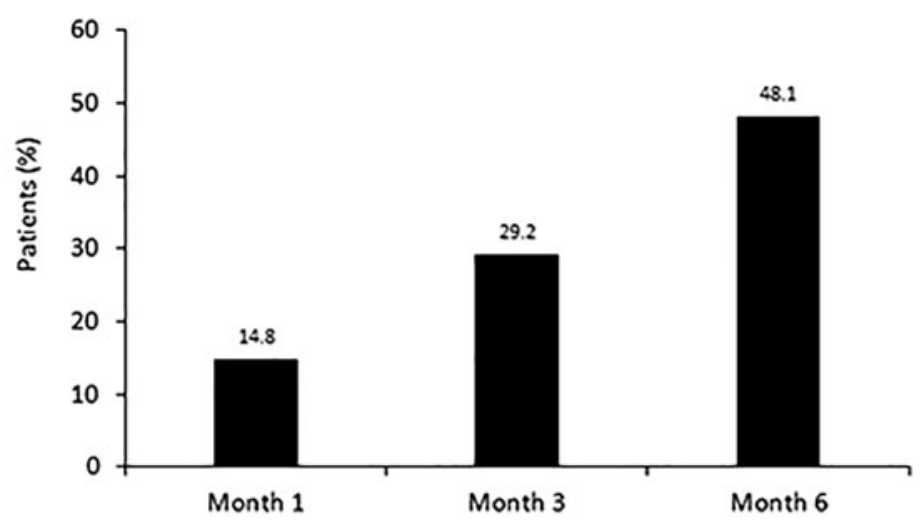

Percentage of patients with at least a 2-grade CFS reduction over time

FIG. 5. (A, B) Corneal healing over time after the instillation of 1 drop of the CE AT in patients with severe DED (CFS $\geq 4$ at the beginning of the study). 
A Symptoms (OSDI) change over time

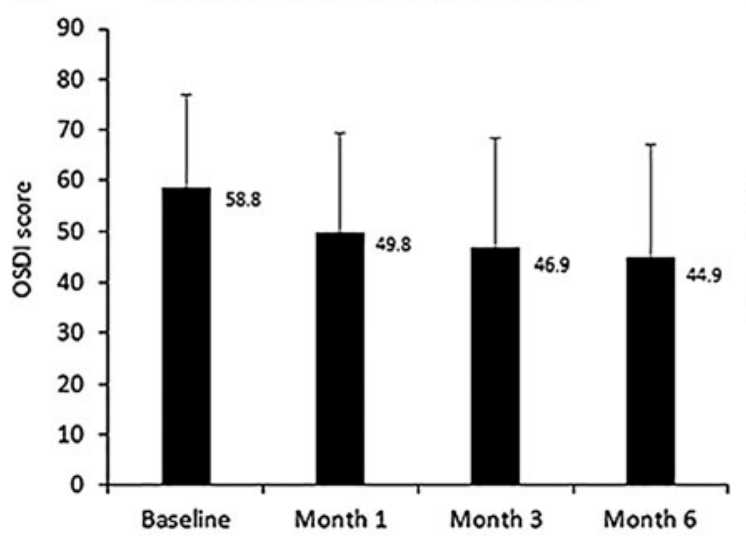

B Patients (\%) experiencing symtpoms most of the time to all of

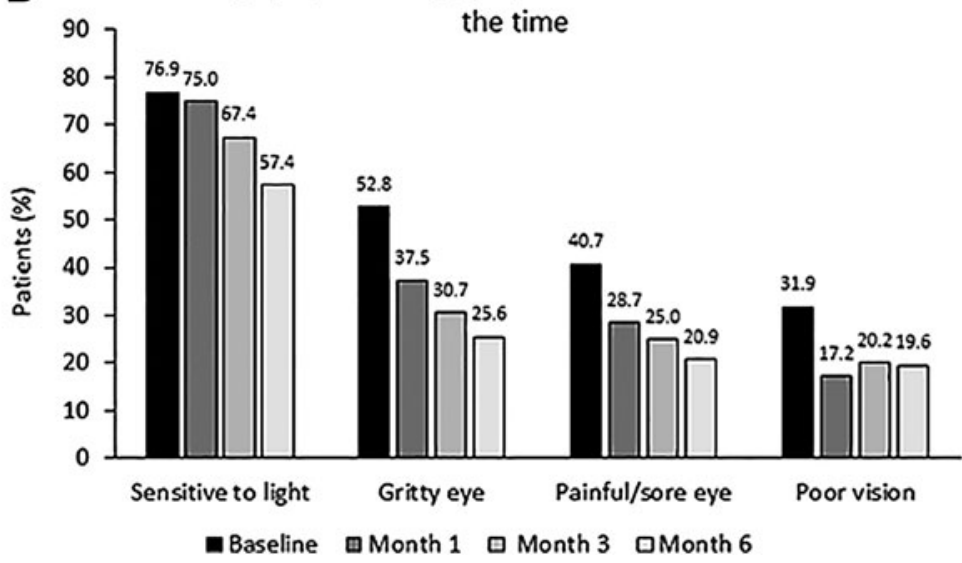

FIG. 6. (A, B) Symptom improvement in a patient population with severe DED on treatment with the CE AT.

\section{Improvement of symptoms with the CES}

Symptoms of DED are of varying severity and were described as stinging, burning or scratchy sensations, foreignbody sensation, increased sensitivity to light, blurred vision, as well as eye fatigue and eye redness. ${ }^{67}$ In DED, ocular surface inflammation, paralleled or not by tissue damage, results in changes in nerve structure and altered responses to external mechanical or chemical stimuli. The peripheral and central sensitization results in a pain response to normally non-painful stimuli. ${ }^{68}$ However, reduced corneal sensitivity is also frequently observed in DE patients, ${ }^{69}$ who often experienced a worsening of their symptoms on improvement of ocular surface tissue damage. ${ }^{70-73}$

Hence, reducing the inflammatory stress of the ocular surface tissue will lead to improvement in the neurosensory abnormality observed in DE patients. It has been reported that CEs harbor some anti-inflammatory properties ${ }^{55,57}$ that result in CFS score improvement in animal models and patients. These improvements in tissue damage were paralleled with an amelioration of symptoms (Fig. 6) in clinical trials. No direct action of CEs on nerves is suspected, and the improvement in the symptoms of DE patients treated with these CEs might instead result from the restoration of a healthy ocular surface environment and state of equilibrium.

\section{Proposed Mechanism of Action of CEs in the Management of DED Signs and Symptoms}

The CEs, through their optimized composition, can help restore the homeostasis of the TF by concomitantly modulating the different causes (identified as the factors (1) to (5) having an etiological role in DED according to DEWS II DE definition) of TF instability, ocular surface inflammation, and tissue damage. Figure 7 is a schematic representation of
FIG. 7. Schematic of the mechanisms of action of CEs in the management of signs and symptoms of DED.

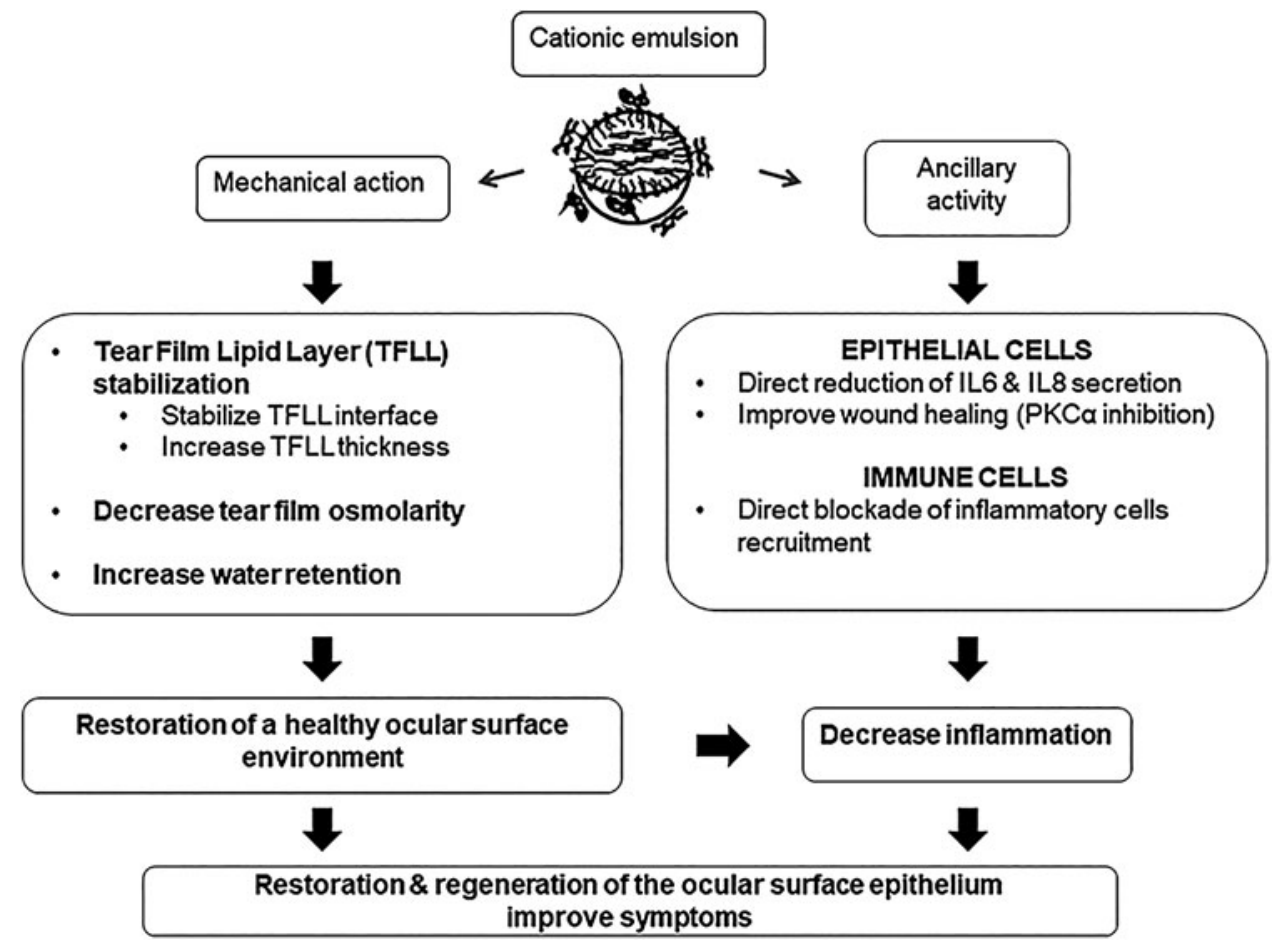


how the concomitant mechanical and pharmacological ancillary actions of the CEs simultaneously improve the signs and symptoms of DE. Table 2 summarizes the contribution of the CE components to the restoration of a healthy state of equilibrium of the ocular surface of DE patients.

\section{Conclusion}

The CEs are a new type of AT that functionally mimic a healthy TF. Indeed, they provide nonpolar and polar lipids to replenish the TFLL and increase its thickness, while simultaneously augmenting the stability of its interface with the aqueous phase of the TF. In addition, the positive charge provided by CKC contributes to the generation of electrostatic forces that help in the adsorption of TF-soluble proteins at the TFLL interface and further stabilize the TFLL. The positive charge of the oil nanodroplets of the CEs also help in the homogeneous spreading of the ATs on the negatively charged ocular surface. The nonionic water-soluble surfactants aid in the stabilization of this interface. The hypo-/iso-osmolarity of the CE ATs also contributes toward the modulation of the hyper-osmolarity of the TF.

Alongside these mechanical effects, the CEs possess ancillary anti-inflammatory properties that contribute to their good performance in DED. Indeed, CKC, by specifically inhibiting $\mathrm{PKC} \alpha$, and tyloxapol, which is able to modulate the action of $\mathrm{NF}-\kappa \mathrm{B}$, reduce the infiltration of inflammatory cells in diseased tissues; decrease the production of proinflammatory cytokines and chemokines (by epithelial and immune cells); and improve the rate of ocular surface healing.

The mechanical properties of the CE eye drops, which represent a functional healthy $\mathrm{TF}$ in a bottle, supported by the ancillary anti-inflammatory properties explain the good performance of CEs in the management of DED signs and symptoms that range from mild to moderate to severe. The contribution of CEs to the restoration of homeostasis of the TF and ocular surface unit in other eye diseases that negatively affect the ocular surface (such as allergy, glaucoma, cataract, and refractive surgeries) should be further investigated.

\section{Acknowledgment}

The authors wish to thank Isabella Athanassiou for her careful review of the article.

\section{Author Disclosure Statement}

P.D., M.A., D.I., J.-S.G.: Santen (E); G.G.: Menicon, Otsuka, Rohto, Santen (F); L.C.: Santen (R); C.B.: Allergan, Laboratoires Thea, Santen (F); Alcon/Novartis, Allergan, Laboratoires Thea, Santen (C); Laboratoires Thea, Dompe Pharma, Santen (R); A.L.: Santen (R); G.G.: none.

\section{Funding Information}

No funding was received for this article.

\section{References}

1. Baudouin, C. The pathology of dry eye. Surv. Ophthalmol. 45 Suppl 2:S211-S220, 2001.

2. Bron, A.J. The Biology of Eye Disease. 2010.

3. Wei, Y., and Asbell, P.A. The core mechanism of dry eye disease is inflammation. Eye Contact Lens. 40:248-256, 2014.
4. Baudouin, C. A new approach for better comprehension of diseases of the ocular surface. J. Fr. Ophtalmol. 30:239246, 2007.

5. Bron, A.J., de Paiva, C.S., Chauhan, S.K., Bonini, S., Gabison, E.E., Jain, S., Knop, E., Markoulli, M., Ogawa, Y., Perez, V., Uchino, Y., Yokoi, N., Zoukhri, D., and Sullivan, D.A. TFOS DEWS II pathophysiology report. Ocul. Surf. 15:438-510, 2017.

6. Yokoi, N., and Georgiev, G.A. Tear film-oriented diagnosis and tear film-oriented therapy for dry eye based on tear film dynamics TFOD and TFOT. Invest. Ophthalmol. Vis. Sci. 59:DES13-DES22, 2018.

7. Tsubota, K., Yokoi, N., Watanabe, H., Dogru, M., Kojima, T., Yamada, M., Kinoshita, S., Kim, H.M., Tchah, H.W., Hyon, J.Y., Yoon, K.C., Seo, K.Y., Sun, X., Chen, W., Liang, L., Li, M., Tong, L., Hu, F.R., Puangsricharern, V., Lim-Bon-Siong, R., Yong, T.K., Liu, Z., Shimazaki, J., and Members of The Asia Dry Eye, Society. A new perspective on dry eye classification: proposal by the Asia Dry Eye Society. Eye Contact Lens. 46 Suppl 1:S2-S13, 2020.

8. Lallemand, F., Daull, P., Benita, S., Buggage, R., and Garrigue, J.S. Successfully improving ocular drug delivery using the cationic nanoemulsion Novasorb. J. Drug Deliv. 2012:604204, 2012.

9. Daull, P., Lallemand, F., and Garrigue, J.S. Benefits of cetalkonium chloride cationic oil-in-water nanoemulsions for topical ophthalmic drug delivery. J. Pharm. Pharmacol. 66:531-541, 2014.

10. Robert, P.-Y., Cochener, B., Amrane, M., Ismail, D., Garrigue, J.-S., Pisella, P.-J., and Baudouin, C. Efficacy and safety of a cationic emulsion in the treatment of moderate to severe dry eye disease: a randomized controlled study. Eur. J. Ophthalmol. 26:546-555, 2016.

11. Leonardi, A., Van Setten, G., Amrane, M., Ismail, D., Garrigue, J.-S., Figueiredo, F.C., and Baudouin, C. Efficacy and safety of $0.1 \%$ cyclosporine A cationic emulsion in the treatment of severe dry eye disease: a multicenter randomized trial. Eur. J. Ophthalmol. 26:287-296, 2016.

12. Hwang, S.B., Park, J.H., Kang, S.S., Kang, D.H., Lee, J.H., Oh, S.J., Lee, J.Y., Kim, J.Y., and Tchah, H. Protective effects of cyclosporine A emulsion versus cyclosporine A cationic emulsion against desiccation stress in human corneal epithelial cells. Cornea. 39:508-513, 2020.

13. Leonardi, A., Doan, S., Amrane, M., Ismail, D., Montero, J., Németh, J., Aragona, P., and Bremond-Gignac, D. A randomized, controlled trial of cyclosporine A cationic emulsion in pediatric vernal keratoconjunctivitis: the VEKTIS Study. Ophthalmology. 126:671-681, $2019 \mathrm{~b}$.

14. Willcox, M.D.P., Argueso, P., Georgiev, G.A., Holopainen, J.M., Laurie, G.W., Millar, T.J., Papas, E.B., Rolland, J.P., Schmidt, T.A., Stahl, U., Suarez, T., Subbaraman, L.N., Ucakhan, O.O., and Jones, L. TFOS DEWS II tear film report. Ocul. Surf. 15:366-403, 2017.

15. Georgiev, G.A., Eftimov, P., and Yokoi, N. Structurefunction relationship of tear film lipid layer: a contemporary perspective. Exp. Eye Res. 163:17-28, 2017.

16. Yokoi, N., Takehisa, Y., and Kinoshita, S. Correlation of tear lipid layer interference patterns with the diagnosis and severity of dry eye. Am. J. Ophthalmol. 122:818-824, 1996.

17. Butovich, I.A. Tear film lipids. Exp. Eye Res. 117:4-27, 2013.

18. Lam, S.M., Tong, L., Duan, X., Petznick, A., Wenk, M.R., and Shui, G. Extensive characterization of human tear fluid collected using different techniques unravels the presence of novel lipid amphiphiles. J. Lipid Res. 55:289-298, 2014. 
19. Brown, S.H., Kunnen, C.M., Duchoslav, E., Dolla, N.K., Kelso, M.J., Papas, E.B., Lazon de la Jara, P., Willcox, M.D., Blanksby, S.J., and Mitchell, T.W. A comparison of patient matched meibum and tear lipidomes. Invest. Ophthalmol. Vis. Sci. 54:7417-7424, 2013.

20. Butovich, I.A. Meibomian glands, meibum, and meibogenesis. Exp. Eye Res. 163:2-16, 2017.

21. Garrigue, J.S., Amrane, M., Faure, M.O., Holopainen, J.M., and Tong, L. Relevance of lipid-based products in the management of dry eye disease. J. Ocul. Pharmacol. Ther. 33:647-661, 2017.

22. Cwiklik, L. Tear film lipid layer: a molecular level view. Biochim. Biophys. Acta. 1858:2421-2430, 2016.

23. Wizert, A., Iskander, D.R., and Cwiklik, L. Organization of lipids in the tear film: a molecular-level view. PLoS One. 9: e92461, 2014.

24. Lam, S.M., Tong, L., Yong, S.S., Li, B., Chaurasia, S.S., Shui, G., and Wenk, M.R. Meibum lipid composition in Asians with dry eye disease. PLoS One. 6:e24339, 2011.

25. Nencheva, Y., Olzynska, A., Melcrova, A., As Georgiev, G., Daull, P., Garrigue, J.-S., and Cwiklik, L. Improving stability of tear film lipid layer via concerted action of two drug molecules: a biophysical view. Biophys. J. 114:104a, 2018.

26. Georgiev, G.A., Yokoi, N., Nencheva, Y., Peev, N., and Daull, P. Surface chemistry interactions of cationorm with films by human meibum and tear film compounds. Int. J. Mol. Sci. 18:E1558, 2017.

27. Georgiev, G.A., Yokoi, N., Ivanova, S., Tonchev, V., and Daull, P. Surface chemistry of the interactions of cationic nanoemulsions with human meibum films. ARVO poster 6188-A0091, Seattle, 2016.

28. Cwiklik, L., Melcrova, A., Daull, P., and Garrigue, J. Tear film break-up: a molecular level view by employing in silico approach. ARVO poster 472-A0397, Baltimore, 2017.

29. Cwiklik, L., Melcrova, A., Daull, P., and Garrigue, J.S. A proposed mechanism for tear film breakup: a molecular level view by employing in-silico approach. J. Model. Ophthalmol. 1:19-23, 2018.

30. Wizert, A., Iskander, D.R., and Cwiklik, L. Interaction of lysozyme with a tear film lipid layer model: a molecular dynamics simulation study. Biochim. Biophys. Acta Biomembr. 1859:2289-2296, 2017.

31. Garhofer, G., Werkmeister, R., Messmer, A., Dos Santos, V., Stegmann, H., Schmidl, D., Fondi, K., and Schmetterer, L. Short-term reproducibility of interferometry based tear film lipid layer thickness measurements in humans. ARVO poster 4398-A0237, Baltimore, 2017.

32. Aranha dos Santos, V., Schmetterer, L., Stegmann, H., Garhofer, G., Schmidl, D., and Werkmeister, R.M. Quantification of the tear film lipid layer using optical coherence tomography. Invest. Ophthalmol. Vis. Sci. 59:271, 2018.

33. Mochizuki, H., Yamada, M., Hatou, S., and Tsubota, K. Turnover rate of tear-film lipid layer determined by fluorophotometry. Br. J. Ophthalmol. 93:1535-1538, 2009.

34. Amrane, M., Creuzot-Garcher, C., Robert, P.Y., Ismail, D., Garrigue, J.S., Pisella, P.J., and Baudouin, C. Ocular tolerability and efficacy of a cationic emulsion in patients with mild to moderate dry eye disease - a randomised comparative study. J. Fr. Ophtalmol. 37:589-598, 2014.

35. Leonardi, A., Messmer, E.M., Labetoulle, M., Amrane, M., Garrigue, J.S., Ismail, D., Sainz-de-la-Maza, M., Figueiredo, F.C., and Baudouin, C. Efficacy and safety of $0.1 \%$ ciclosporin A cationic emulsion in dry eye disease: a pooled analysis of two double-masked, randomised, vehicle-controlled phase III clinical studies. Br. J. Ophthalmol. 103:125-131, 2019a.

36. Baudouin, C., Aragona, P., Messmer, E.M., Tomlinson, A., Calonge, M., Boboridis, K.G., Akova, Y.A., Geerling, G., Labetoulle, M., and Rolando, M. Role of hyperosmolarity in the pathogenesis and management of dry eye disease: proceedings of the OCEAN group meeting. Ocul. Surf. 11: 246-258, 2013.

37. Lemp, M.A., Bron, A.J., Baudouin, C., Del Castillo, J.M., Geffen, D., Tauber, J., Foulks, G.N., Pepose, J.S., and Sullivan, B.D. Tear osmolarity in the diagnosis and management of dry eye disease. Am. J. Ophthalmol. 151:792798.e1, 2011.

38. Li, D.Q., Chen, Z., Song, X.J., Luo, L., and Pflugfelder, S.C. Stimulation of matrix metalloproteinases by hyperosmolarity via a JNK pathway in human corneal epithelial cells. Invest. Ophthalmol. Vis. Sci. 45:4302-4311, 2004.

39. Luo, L., Li, D.Q., Corrales, R.M., and Pflugfelder, S.C. Hyperosmolar saline is a proinflammatory stress on the mouse ocular surface. Eye Contact Lens. 31:186-193, 2005.

40. Potvin, R., Makari, S., and Rapuano, C.J. Tear film osmolarity and dry eye disease: a review of the literature. Clin. Ophthalmol. 9:2039-2047, 2015.

41. Craig, J.P., Nelson, J.D., Azar, D.T., Belmonte, C., Bron, A.J., Chauhan, S.K., de Paiva, C.S., Gomes, J.A.P., Hammitt, K.M., Jones, L., Nichols, J.J., Nichols, K.K., Novack, G.D., Stapleton, F.J., Willcox MDP, Wolffsohn, J.S., and Sullivan, D.A. TFOS DEWS II report executive summary. Ocul. Surf. 15:802-812, 2017.

42. Suzuki, M., Massingale, M.L., Ye, F., Godbold, J., Elfassy, T., Vallabhajosyula, M., and Asbell, P.A. Tear osmolarity as a biomarker for dry eye disease severity. Invest. Ophthalmol. Vis. Sci. 51:4557-4561, 2010.

43. Gilbard, J.P. Human tear film electrolyte concentrations in health and dry-eye disease. Int. Ophthalmol. Clin. 34:2736, 1994.

44. Beck, R., Stachs, O., Koschmieder, A., Mueller-Lierheim Wolfgang, G.K., Peschel, S., and van Setten, G.-B. Hyaluronic acid as an alternative to autologous human serum eye drops: initial clinical results with high-molecularweight hyaluronic acid eye drops. Case Rep. Ophthalmol. 10:244-255, 2019.

45. Holly, F.J., and Lamberts, D.W. Effect of nonisotonic solutions on tear film osmolality. Invest. Ophthalmol. Vis. Sci. 20:236-245, 1981.

46. Corrales, R.M., Luo, L., Chang, E.Y., and Pflugfelder, S.C. Effects of osmoprotectants on hyperosmolar stress in cultured human corneal epithelial cells. Cornea. 27:574-579, 2008.

47. Chen, W., Zhang, X., Li, J., Wang, Y., Chen, Q., Hou, C., and Garrett, Q. Efficacy of osmoprotectants on prevention and treatment of murine dry eye. Invest. Ophthalmol. Vis. Sci. 54:6287-6297, 2013.

48. van Setten, G.B. Osmokinetics: a new dynamic concept in dry eye disease. J. Fr. Ophtalmol. 42:221-225, 2019.

49. Simmons, P., Chang-Lin, J.-E., Chung, Q., Vehige, J., and Welty, D. Effect of compatible solutes on transepithelial electrical resistance and uptake in primary rabbit corneal epithelial cell layers model. Invest. Ophthalmol. Vis. Sci. 48:428, 2007.

50. Warcoin, E., Clouzeau, C., Roubeix, C., Raveu, A.L., Godefroy, D., Riancho, L., Baudouin, C., and BrignoleBaudouin, F. Hyperosmolarity and benzalkonium chloride differently stimulate inflammatory markers in conjunctiva- 
derived epithelial cells in vitro. Ophthalmic Res. 58:40-48, 2017.

51. Bron, A.J., Evans, V.E., and Smith, J.A. Grading of corneal and conjunctival staining in the context of other dry eye tests. Cornea. 22:640-650, 2003.

52. Daull, P., Feraille, L., Barabino, S., Cimbolini, N., Antonelli, S., Mauro, V., and Garrigue, J.S. Efficacy of a new topical cationic emulsion of cyclosporine A on dry eye clinical signs in an experimental mouse model of dry eye. Exp. Eye Res. 153:159-164, 2016.

53. Quentric, Y., Daull, P., Feraille, L., Elena, P.P., and Garrigue, J.S. Efficacy of a preservative-free cationic emulsion vehicle eye drop in a mouse model of dry eye. ARVO poster 422-D0131, Seattle, 2016.

54. Liang, H., Baudouin, C., Daull, P., Garrigue, J.S., and Brignole-Baudouin, F. In vitro and in vivo evaluation of a preservative-free cationic emulsion of latanoprost in corneal wound healing models. Cornea. 31:1319-1329, 2012.

55. Daull, P., Feraille, L., Elena, P.P., and Garrigue, J.S. Comparison of the anti-inflammatory effects of artificial tears in a rat model of corneal scraping. J. Ocul. Pharmacol. Ther. 32:109-118, 2016.

56. Daull, P., Guenin, S., and Garrigue, J.-S. Mechanism of action of cationic emulsions in the management of ocular surface inflammation and wound healing. ARVO poster 5053-B0259, Seattle, 2016.

57. Daull, P., Guenin, S., Hamon de Almeida, V., and Garrigue, J.S. Anti-inflammatory activity of CKC-containing cationic emulsion eye drop vehicles. Mol. Vis. 24:459-470, 2018.

58. Liang, H., Brignole-Baudouin, F., Rabinovich-Guilatt, L., Mao, Z., Riancho, L., Faure, M.O., Warnet, J.M., Lambert, G., and Baudouin, C. Reduction of quaternary ammoniuminduced ocular surface toxicity by emulsions: an in vivo study in rabbits. Mol. Vis. 14:204-216, 2008.

59. Liang, H., Baudouin, C., Faure, M.O., Lambert, G., and Brignole-Baudouin, F. Comparison of the ocular tolerability of a latanoprost cationic emulsion versus conventional formulations of prostaglandins: an in vivo toxicity assay. Mol. Vis. 15:1690-1699, 2009.

60. Liang, H., Baudouin, C., Daull, P., Garrigue, J.S., and Brignole-Baudouin, F. Ocular safety of cationic emulsion of cyclosporine in an in vitro corneal wound healing model and an acute in vivo rabbit model. Mol. Vis. 18:2195-2204, 2012.

61. Daull, P., Raymond, E., Feraille, L., and Garrigue, J.S. Safety and tolerability of overdosed artificial tears by abraded rabbit corneas. J. Ocul. Pharmacol. Ther. 34:670676, 2018.

62. Chen, Z., Li, Z., Basti, S., Farley, W.J., and Pflugfelder, S.C. Altered morphology and function of the lacrimal functional unit in protein kinase $\mathrm{C}\{$ alpha $\}$ knockout mice. Invest. Ophthalmol. Vis. Sci. 51:5592-5600, 2010.

63. Antal, J.M., Divis, L.T., Erzurum, S.C., Wiedemann, H.P., and Thomassen, M.J. Surfactant suppresses NF-kappa B activation in human monocytic cells. Am. J. Respir. Cell Mol. Biol. 14:374-379, 1996.
64. Thomassen, M.J., Antal, J.M., Divis, L.T., and Wiedemann, H.P. Regulation of human alveolar macrophage inflammatory cytokines by tyloxapol: a component of the synthetic surfactant Exosurf. Clin. Immunol. Immunopathol. 77:201-205, 1995.

65. Kuo, J.H., Lin, Y.L., and Tseng, J.W. Interactions between U-937 human macrophages and tyloxapol. Colloids Surf. B Biointerfaces. 64:208-215, 2008.

66. Wolffsohn, J.S., Arita, R., Chalmers, R., Djalilian, A., Dogru, M., Dumbleton, K., Gupta, P.K., Karpecki, P., Lazreg, S., Pult, H., Sullivan, B.D., Tomlinson, A., Tong, L., Villani, E., Yoon, K.C., Jones, L., and Craig, J.P. TFOS DEWS II Diagnostic Methodology report. Ocul. Surf. 15: 539-574, 2017.

67. Labetoulle, M., Rolando, M., Baudouin, C., and van Setten, G. Patients' perception of DED and its relation with time to diagnosis and quality of life: an international and multilingual survey. Br. J. Ophthalmol. 101:1100-1105, 2017.

68. Tuisku, I.S., Konttinen, Y.T., Konttinen, L.M., and Tervo, T.M. Alterations in corneal sensitivity and nerve morphology in patients with primary Sjogren's syndrome. Exp. Eye Res. 86:879-885, 2008.

69. Bron, A.J., Yokoi, N., Gafney, E., and Tiffany, J.M. Predicted phenotypes of dry eye: proposed consequences of its natural history. Ocul. Surf. 7:78-92, 2009.

70. Benitez-Del-Castillo, J.M., Acosta, M.C., Wassfi, M.A., Diaz-Valle, D., Gegundez, J.A., Fernandez, C., and GarciaSanchez, J. Relation between corneal innervation with confocal microscopy and corneal sensitivity with noncontact esthesiometry in patients with dry eye. Invest. Ophthalmol. Vis. Sci. 48:173-181, 2007.

71. Xu, K.P., Yagi, Y., and Tsubota, K. Decrease in corneal sensitivity and change in tear function in dry eye. Cornea. 15:235-239, 1996.

72. Labbe, A., Liang, Q., Zhang, Y., Wang, Z., Xu, L., Baudouin, C., and Sun, X. Corneal nerve structure and function in patients with non-Sjogren dry eye: clinical correlations. Invest. Ophthalmol. Vis. Sci. 54:5144-5150, 2013.

73. Adatia, F.A., Michaeli-Cohen, A., Naor, J., Caffery, B., Bookman, A., and Slomovic, A. Correlation between corneal sensitivity, subjective dry eye symptoms and corneal staining in Sjogren's syndrome. Can. J. Ophthalmol. 39: 767-771, 2004.

Received: February 3, 2020 Accepted: April 19, 2020

Address correspondence to: Dr. Jean-Sebastien Garrigue Novagali Innovation Center Santen SAS

1 rue Pierre Fontaine, bât. Genavenir IV

Evry 91000

France

E-mail: jean-sebastien.garrigue@santen.com 